\title{
DEMOCRACIA Y EDUCACIÓN SUPERIOR: UN RECORRIDO POR LAS PUBLICACIONES DE LA UNIVERSIDAD NACIONAL DE ROSARIO (1986-1990)
}

\author{
María del Carmen Fernández (Universidad Nacional de Rosario)* \\ mcfer7@gmail.com
}

Recibido: 30/08/13 Aceptado: 15/11/13

\section{Resumen}

En diciembre de 1983 se inició en la Argentina una nueva coyuntura política, conocida como "la transición democrática". Durante ese período se llevaron a cabo una serie de medidas en defensa de un sistema democrático que se reconocía vulnerable. Una de las tareas fundamentales consistió en comenzar a desmantelar un orden autoritario a partir de la transmisión de valores democráticos, tarea que llevó adelante fundamentalmente el sistema educativo a través de sus distintos niveles. En ese sentido, las Universidades asumieron un fuerte compromiso político.

En este artículo se analiza la política editorial, referida a educación, llevada adelante por la Universidad Nacional de Rosario durante 1986-1990. La misma consistió en la publicación de una serie de colecciones con el propósito de contribuir al proceso de democratización en los claustros, las instituciones, la vida académica y la universidad en su conjunto.

\section{Palabras claves}

Orden Autoritario - Democracia - Sistema Educativo - Política Universitaria Publicaciones Académicas.

* Magíster en Educación con Mención en Historia y Prospectiva (UNER). Diploma en Ciencia Política, FLACSO Rosario. Lic. en Ciencia Política (UNR). Prof. en Ciencias de la Educación (UNR). Prof. Adjunta Núcleo Histórico Epistemológico de la Educación (Prof. y Lic. en Ciencias de la Educación; Historia Socio Política del Sistema Educativo Argentino, Formación Docente). Facultad de Humanidades y Artes, UNR. Miembro de la Comisión Directiva de la Sociedad Argentina de Historia de la Educación. 


\begin{abstract}
In December 1983 began a new political situation in Argentina, known as "democratic transition". During this period, a series of measures were carried out in a democratic system that it recognized vulnerable. The main task was to dismantle authoritarian order from the transmission of democratic values, task that was carried on primarily the education system through various levels. In that regard, the universities assumed a strong political commitment. This article discusses the editorial policy run by the National University of Rosario in 1986-1990. This policy was the publication of a series of collections with the purpose of contributing to the process of democratization in the cloisters, the institutions, the academic life and the University as a whole.
\end{abstract}

\title{
Key words
}

Authoritarian order - democracy - education system - university politics academic publications.

\section{Transición democrática y educación superior}

El 24 de marzo de 1976 las Fuerzas Armadas asumieron el gobierno en la República Argentina e inauguraron una dictadura que se sostuvo en el poder a partir de la suspensión de las garantías individuales, la sistematización de la persecución política y la imposición del terrorismo de Estado. Esta dictadura, autodenominada "Proceso de Reorganización Nacional", llevó adelante una feroz represión sin precedentes en el país y fue la responsable de la desaparición, encarcelamiento y exilio de miles de ciudadanos, entre los cuales se contaron numerosos miembros de la comunidad universitaria. Las universidades sufrieron el control policial que invadió sus espacios, la imposición de perspectivas teóricas alineadas con el régimen, el desmantelamiento de sus órganos de gobierno y el terror que imponen las decisiones arbitrarias de gobernantes impunes. La Universidad Nacional de Rosario no fue una excepción en esta política de avasallamiento.

El gobierno de Raúl Alfonsín en diciembre de 1983 inicia un nuevo proceso denominado "de transición democrática". Un año antes Francisco Delich (1982) uno de los autores que más explícitamente utilizó el término "transición", lo empleó para referirse a un periodo caracterizado por una fuerte ambigüedad, que deviene de la coexistencia de elementos de cada uno de los dos polos entre los cuales se transita: el polo autoritario y el polo democrático. Waldo Ansaldi (2006) realiza un análisis más complejo al referirse a la noción de transición, señalando que se trata de un proceso de duración variable, iniciado todavía en situación de dictadura y generado por distintas razones: protestas populares, acciones concertadas entre los partidos políticos o fracturas dentro de las Fuerzas Armadas y que culmina cuando el nuevo gobierno democrático no posee ningún tipo de condicionantes ni cuestionamientos originados en el 
ejercicio de la dictadura e impuestos por la misma en momentos de su retirada de la escena política.

El nuevo gobierno democrático, tanto por la herencia del periodo autoritario como por sus propias limitaciones, vio condicionada su capacidad de revertir la crisis económica de un modelo de Estado que se había iniciado una década atrás. Con respecto al orden político, además de los intentos de lograr una "concertación" con los distintos actores sociales, políticos y económicos, su principal preocupación se centró en la necesidad de transformar una cultura autoritaria. Parte de este diagnóstico, siguiendo a O'Donell (1982) estaba vinculado con la capacidad que tuvo el gobierno militar de penetrar "capilarmente" la sociedad mediante valores y pautas de comportamiento autoritarias. Los intentos de desestabilización del sistema institucional por parte de las Fuerzas Armadas durante este periodo mostraron que era necesario mantener presente la importancia de la defensa del sistema democrático. En consonancia con este objetivo, y habiendo sido el sistema educativo uno de los principales instrumentos para la afirmación de las concepciones autoritarias, la función principal de la educación estuvo dirigida a desmantelar el orden autoritario a partir de la transmisión de valores democráticos.

En uno de los documentos elaborados por el nuevo gobierno se sintetiza el papel de la educación en la construcción de una sociedad democrática:

Dentro de los objetivos globales del presente gobierno debe buscarse por todos los medios que nuestra educación haga un aporte al afianzamiento y desarrollo de la democratización del país, lo que difícilmente pueda lograrse sin democratizar las prácticas, contenidos, métodos y organización de la educación misma (1).

El citado Documento plantea tres objetivos fundamentales: garantizar el acceso permanencia y reinserción de la población en todos los niveles del sistema educativo; fomentar la participación de la comunidad educativa desarrollando canales adecuados de participación en las diferentes instancias del sistema educativo; proporcionar a los alumnos un servicio educativo con procesos de enseñanza-aprendizaje que estimulen la creatividad, el espíritu crítico, con la adecuación de los contenidos, planes de estudio y metodologías de enseñanza de acuerdo a las contribuciones de la pedagogía y de otras ciencias y a los requerimientos culturales, sociales y económicos de la realidad nacional y regional.

La preocupación por la instauración de la democracia en todas las instituciones y por los cambios que la misma generaría en todos los aspectos de la sociedad marcó el clima de época. Esa visión también estuvo presente en la universidad que fue uno de los bastiones políticos del partido gobernante.

Durante el proceso de recuperación de la democracia, las universidades tuvieron un rol muy importante. La comunidad universitaria, a través de la asunción de compromisos políticos y la organización partidaria y extrapartidaria 
de las agrupaciones estudiantiles, apoyó la apertura democrática con distintas acciones que abarcaron desde marchas multitudinarias en defensa de la democracia hasta la creación de espacios de reflexión respecto de qué modelo de sociedad se propiciaría. La condena de los crímenes de la dictadura fue unánime y la democracia volvió a las universidades al mismo tiempo que se gestaban los Juicios a las Juntas y el Congreso Pedagógico Nacional.

Las nuevas autoridades manifestaron su voluntad de restablecer la democracia en las universidades, reconstruir los claustros docentes a partir de los concursos y asegurar la participación de estudiantes y graduados en el gobierno universitario; suprimir los exámenes de ingreso y el arancelamiento; fomentar la investigación científica; solucionar los problemas edilicios; reincorporar a los docentes cesanteados en el periodo anterior; poner fin a la discriminación ideológica; organizar instancias de formación docente acordes con los nuevos lineamientos políticos y con la actualización de conocimientos y avances científicos; modificar las modalidades de enseñanza vigentes en la etapa anterior; autorizar a las universidades a administrar sus propios recursos; revisar y reformular planes y programas de estudios; impulsar medidas tendientes a resolver aquellos problemas derivados de la explosión de la matrícula; establecer una nueva relación entre Estado y Universidad de manera de asegurar la vigencia de la autonomía; etc.

En diciembre de 1983 se intervinieron las universidades, se dispuso que comenzaran a funcionar sobre la base de los estatutos que regían en 1966 y se les otorgó un año de plazo para la normalización. Como en 1966 la Universidad Nacional de Rosario aún no había sido creada, se estableció que se regiría por el Estatuto de la Universidad Nacional del Litoral hasta tanto dictara el propio (2). Finalmente se llamó a elecciones en el mes de abril de 1986.

\section{La Universidad Nacional de Rosario y su compromiso con la democracia}

La Universidad Nacional de Rosario se creó por Ley 17987 el 29 de noviembre de 1968, como un desprendimiento de la Universidad Nacional del Litoral.

En el período que nos ocupa por primera vez normaliza su gobierno y en mayo de 1986 asume el Dr. Juan Carlos Millet, primer Rector electo por la Asamblea Universitaria.

En su primer discurso, el Rector Millet expresa:

(...) Una universidad que privilegie el desarrollo de conocimientos debe partir de la necesidad de erradicar hasta los cimientos el autoritarismo que ha anidado a lo largo de décadas. Esta lucha contra el autoritarismo la debemos desarrollar en todo momento y en todos los niveles. Para ello es necesaria la participación real y efectiva de todos los ciudadanos universitarios (docentes, estudiantes, graduados y no-docentes). Debemos, como bien afirmamos en nuestro 
programa, realizar un vuelco copernicano del concepto de alumno, estableciendo una centralidad fundamental del mismo, pasando de pensar al docente como el depositario del saber y concibiendo al alumno como el lugar donde el saber debe ser puesto, a pensar a este último no como un objeto al que debemos modelar sino como un sujeto activo y responsable de su propia formación. Sólo concibiendo a la clase como una instancia de producción cooperativa, en donde tanto el docente como el alumno conforman un equipo de producción de conocimientos, podremos establecer la posibilidad de la creación colectiva dentro de los claustros y por lo tanto su participación real y efectiva. Esta concepción presupone desarrollar un nuevo concepto de lo que es y cómo funciona una cátedra universitaria. Ordinariamente se ha entendido la cátedra como conformada por los docentes que actúan en ella en el mejor de los casos, o como una propiedad del profesor titular quien tiene el derecho de establecer su voluntad omnímoda dentro de lo que tradicionalmente se ha entendido como cátedra feudo. Creemos que debemos entender bajo el concepto de cátedra al conjunto del grupo de trabajo que conforman docentes y alumnos, y precisar su actividad como una tarea colectiva de producción y aprendizaje. (...) No se nos escapa la necesidad de contar con un fondo editorial propio que posibilite el desarrollo de un servicio tan fundamental para la actividad universitaria como lo es el de publicaciones. En ese sentido, entre los proyectos más inmediatos podemos citar el de la creación de la Editorial Universitaria de Rosario y otros proyectos de desarrollo cultural como lo son la creación de un canal y una radio universitaria (3).

Este fragmento del discurso resulta relevante por afirmar la pronta creación de la Editorial Universitaria y por presentar un conjunto de concepciones referidas a la educación y enseñanza universitaria que más tarde serían desarrolladas y fundamentadas en las distintas publicaciones de la propia universidad.

\section{La Editorial Universitaria y las colecciones educativas}

En 1968 cuando se crea la Universidad Nacional de Rosario comienza a funcionar un modesto Servicio de Publicaciones destinado a la impresión de papelería y folletería. Este Servicio empieza su etapa de expansión a partir de 1986 con la recuperación democrática. Se crea entonces, tal como lo había expresado el Rector en su primer discurso, la Dirección de Publicaciones dependiente de la Secretaría de Extensión Universitaria. Sobre esa base en 1989 se crea la Editorial Universitaria y en 1994 la actual UNR Editora.

Teniendo en cuenta los "Lineamientos" elaborados por el Ministerio, la importancia otorgada a la relación educación/democracia por parte del Rector y con el objetivo de modificar/replantear los andamiajes institucionales así como las propuestas curriculares y didácticas heredados, la Secretaría Académica 
comienza a publicar a través de la entonces Dirección de Publicaciones distintas colecciones que se distribuían en todas las Facultades y unidades académicas.

Las colecciones que abordaron con mayor especificidad temas educativos fueron las siguientes:

Colección Cuadernos de Psicología y Psicoanálisis conformada por once cuadernillos: Jaitin, R., Fundamentos de una psicología educacional; López, J., El sujeto en el campo del psicoanálisis; Ageno, R., Apuntes para una psicología de la educación; Emmanuele, E. y Otros, Inserción del psicólogo en educación; Baraldi, C., El sujeto y las vicisitudes de sus aprendizajes, una lectura posible; Ageno, R., La problemática del aprendizaje. Del tiempo cronológico al tiempo lógico; Álvarez, A., Beretta, A. y Ritvo, J.; Epistemología y Psicoanálisis. Psicoanálisis y ciencia: la especulación freudiana; Rosbaco, I., Breve historia de los aportes de la psicología en educación; Baraldi, C., Qué es un niño. Aportes de introducción al psicoanálisis; Inserción del psicólogo en educación; Ageno, R., La didáctica de la matemática. Sus bases psicológicas; Garay, G., Casos de clínica.

Colección Cuadernos de Formación Docente compuesta por doce cuadernillos: Achilli, E., La práctica docente: una interpretación desde los saberes del maestro; Ageno, R.; Apuntes para una psicología de la educación; Monestés, M. C., Fundamentos epistemológicos y proceso educativo; Achilli, E., Ageno, R. y Ossanna, E., Investigación en la práctica docente en taller de educadores; Batallán, G. y García, J., Problemas de la investigación participante y la transformación de la escuela; Emmanuele, E., Universidad, docencia y saber pedagógico; Vera, R., Orientaciones básicas de los talleres de educadores; Bella de Paz, L., Una propuesta curricular alternativa en la Universidad; Ageno, R., El taller de educadores y la investigación; Achilli, E., Escuela y democratización; Brovelli, M., Aportes acerca del problema de la didáctica; Avendaño, F.; El problema de la enseñanza en las ciencias del lenguaje.

Colección Espacios compuesta por cinco cuadernillos: Emmanuele, E. y Otros., La pedagogía universitaria, hoy; Sanjurjo, L. y Boffa, S., La asesoría pedagógica. Su espacio en el ámbito universitario; Emmanuele, E. y Otros, Aportes para una reformulación académica; Sanjurjo, L., Ascolani, A. y Emmanuele, E., La práctica educativa, lecturas posibles; Monestés, M. C., Epistemología y educación.

Colección Propuestas con cinco cuadernillos: Gaitini, A. y Otros, Reflexiones sobre la práctica docente; Avendaño, F. y Otros, Problemática del lenguaje, la literatura y el discurso; Godoy, C., La Historia-problema. Una alternativa de cambio para el aprendizaje de la historia; De Juano, M. y Otros, Como leer; Achilli, E., Profe...¿ ¿No quiere que cambiemos la escuela?

Colección Cuadernos de Ciencias Sociales con nueve cuadernillos. Contardi, S. y Otros, Cultura política y proclamas militares (1930-1976); Levín, S., La seguridad social como sistema; Martínez de Aguirre, E. y Siganecich, P., 
Investigación y discurso social; Achilli, E., Democratización escolar. Estudio antropológico; Ossanna, E. y Ascolani, A., La historia de la educación en la Argentina; Quiroga, H., Marx y la teoría del Estado; Escudero, L. La estructura de la comunicación política; Caponi, S. y Capón, G., Estudios histórico-epistemológicos; Batallán, G. y García, J. Cuestiones de métodos en Ciencias Sociales.

Estos cuadernillos formaron parte de la bibliografía incluida en los programas de las asignaturas y de otros espacios curriculares de distintas carreras de la universidad, fundamentalmente en Ciencias de la Educación y Formación Docente. En cuanto a los autores de estas publicaciones, un gran porcentaje fueron profesores que la dictadura había dejado prescindibles por no reunir las condiciones de "garantía ideológica" y que en esta nueva etapa se reintegran al ámbito académico. En relación a los temas abordados, algunos habían sido eliminados durante el periodo autoritario tanto de los planes de estudio como de los programas de las materias, por considerar que atentaban contra el orden y el estilo de vida "occidental y cristiano" que la dictadura intentó imponer.

Durante esta etapa las universidades además de recuperar los mecanismos de participación que habían sido clausurados, incorporaron contenidos que habían sido objeto de censura así como temáticas, autores, investigaciones y producciones recientes que habían permanecido ausentes en los ámbitos académicos.

Estas colecciones dan cuenta de ese contexto. En ellas se publicaron temas y abordajes que se esperaba contribuyeran al proceso de democratización en los claustros, las instituciones, la vida académica, la universidad en su conjunto. De la variedad de temáticas se han seleccionado cuatro, para analizarlas más detenidamente, por entender que desde distintos enfoques y a través de distintas modalidades se reiteran; porque fueron relevantes en tanto promovieron la democratización de la educación superior y porque formaron parte del debate pedagógico de la época.

\section{El Taller como opción metodológica y su relación con el rol docente.}

La dictadura impuso un orden autoritario en el ámbito educativo basado en la obediencia y el respeto absoluto a la autoridad del docente, quien a su vez debía acatar las disposiciones emanadas de los directivos, supervisores, etc. De este modo la lógica militar penetró en el conjunto del sistema educativo generando disciplinamientos, silencios, parálisis, etc.

Para renovar las culturas institucionales en el marco de un nuevo orden democrático resultaba imprescindible desarticular los mecanismos autoritarios, generar instancias de participación, promover dispositivos donde los actores pudiesen recuperar la palabra, democratizar relaciones y vínculos, etc. Estos atributos, propios de un régimen democrático, hubo que restablecerlos en los distintos planos y aspectos de la realidad social. Y el ámbito educativo, específicamente el nivel superior universitario, no fue una excepción. 
"Recrear las condiciones para la convivencia democrática constituyó una consigna central de quienes asumieron por entonces la conducción de las instituciones universitarias" (Buchninder, 2005: 216). La instauración del nuevo orden requería en el ámbito educativo en general y en la universidad en particular democratizar los vínculos institucionales y transformar el rol docente, convirtiéndolo en un agente de cambio educativo y social. Para ello se necesitaba crear un andamiaje teórico-práctico a partir del cual los docentes pudiesen reflexionar críticamente sobre su propia práctica y buscar alternativas de acción. La opción metodológica fue el Taller. Cabe mencionar que el Taller se convirtió durante esos años en la propuesta de trabajo, de perfeccionamiento y de investigación más difundida, en la medida que se trataba de una instancia participativa en la cual sus integrantes al mismo tiempo que recuperaban la palabra, investigaban y realizaban producciones grupales.

El Taller -como método de perfeccionamiento- se propone ser una modalidad de reorganización del trabajo docente al interior de los establecimientos educativos de manera tal de ir convirtiendo este trabajo altamente burocratizado en un trabajo asumido grupalmente por los propios profesores (Vera, 1987: 2).

De este modo, en las instituciones educativas que optaron por esta propuesta metodológica, los Talleres se integraron a la jornada de trabajo y funcionaron de manera autogestionaria. Su mérito fue permitir que el grupo de profesores reflexionara sobre su propia práctica con la finalidad de poder modificarla y contribuir al cambio educacional, fundamentalmente a la democratización de los modos de enseñar y aprender y de relacionarse en las instituciones.

El Taller es concebido como una alternativa de organización del trabajo docente apta para impulsar un mejoramiento y cambio a partir de la modificación de los propios actores y de su práctica en una perspectiva global de transformación de los sistemas escolares (Vera, 1987: 6).

El Taller constituye una propuesta alternativa a las instancias de capacitación conocidas, en tanto la capacitación es concebida como una investigación acerca de la práctica pedagógica, entendiendo como tal no un hecho individual y aislado, sino como un proceso que se desarrolla en contexto áulico en el cual se establece una particular relación entre el docente, el conocimiento y los alumnos y donde se ponen en juegos dos tareas, enseñar y aprender. El riesgo es que el docente no le otorgue sentido a la unidad de todo el proceso, a la construcción del conocimiento y su recreación con los alumnos.

Frente a la herencia educativa de la dictadura (un sistema escolar autoritario, burocrático, dogmático y disciplinado) se tornó indispensable propiciar cambios que permitieran la democratización a partir del protagonismo de los propios actores, entendiendo que el cambio se produce primero en pequeña 
escala (aula, escuela) para luego llegar lentamente a todos los intersticios del sistema. En este sentido, el Taller al permitir que los educadores participantes recuperen críticamente su saber se constituía en un instrumento facilitador del cambio. "Para que la integración del docente sea crítica, el Taller tiene que actuar como develador de nudos problemáticos o enajenantes presentes en su práctica, en sus representaciones y en su relación con el conocimiento" (Achilli, 1987: 17). En realidad el objetivo a largo plazo consistía en lograr que los docentes pudiesen participar en la elaboración, gestión y control de políticas educativas a través de proyectos pedagógicos democráticos y creativos, convirtiéndose en una alternativa a las políticas heredadas (4).

\section{Pedagogía y Universidad}

El nivel superior universitario fue el que más sufrió los embates de la dictadura: desaparición y cesantías de alumnos y docentes, cierre de algunas carreras, modificación oscurantista de los planes de estudios, censura de contenidos, ingreso restricto, arancelamiento, etc.

Debido a ello, una vez recuperada la democracia y comenzado el proceso de normalización universitaria, la propia universidad se convirtió en objeto de estudio. En el caso de la Universidad Nacional de Rosario y de las publicaciones que nos ocupan, se le dedica un espacio importante a la nueva situación. El incremento exponencial de ingresantes y la nueva coyuntura genera interrogantes acerca del rol que debe cumplir la universidad en una sociedad democrática, tanto en términos de formación de los alumnos como de su articulación con un proyecto de país diferente. Es en ese punto donde se advierte la importancia de la práctica educativa universitaria y de la producción de conocimiento científico. En los artículos de las distintas colecciones reiteradamente se alude a la "pedagogía universitaria" y a la importancia que se le debe otorgar a la formación pedagógica de los profesores, teniendo en cuenta que tradicionalmente el nivel superior ha priorizado la formación teórica o especializada en detrimento de los modos de enseñar, generando cátedras-feudos que tienden a fragmentar el conocimiento. "En la cotidianeidad del quehacer educativo, las dicotomías entre teoría y práctica; aprendizaje y evaluación; producción y transmisión de conocimiento; investigación y docencia; docencia universitaria y ejercicio profesional, constituyen verdaderos divorcios, cuya vigencia resulta muy difícil de revertir" (Emmanuele y Bertolano, 1988: 8).

Para poner fin a algunas de las dicotomías mencionadas y entendiendo a la educación como una práctica social a partir de la cual se podían revertir algunas herencias autoritarias que aún estaban presentes, se plantea la responsabilidad que la propia universidad tiene de revisar la formación de sus docentes. Además de nuevos dispositivos de formación y perfeccionamiento, se crean Asesorías Pedagógicas en las distintas unidades académicas, concibiéndolas como espacios pedagógicos, no aislados, sino integrados a la vida y dinámica 
institucional. Por ese motivo la Asesoría Pedagógica debía estar en estrecha vinculación con la Secretaría Académica de cada Facultad, en tanto le brinda una apoyatura importante.

Ahora bien, para comprender de este modo a la Asesoría Pedagógica, resulta imprescindible concebir al asesor pedagógico desde una nueva lógica. Desde una perspectiva tradicional el asesor pedagógico fue visto como un técnico o un especialista dedicado a resolver problemas entre el profesor y los alumnos, en contextos donde las cátedras se organizan de forma piramidal y jerárquica con la finalidad de transmitir conocimientos acabados. Desde una perspectiva participativa y democrática, las cátedras se convierten en instancias de discusión y de producción de un conocimiento provisorio, el rol del asesor pedagógico se modifica sustancialmente.

En estas publicaciones se evidencia la íntima y estrecha relación que los autores establecen entre Pedagogía Universitaria, Asesoría Pedagógica y Proyecto Político. En este sentido advierten acerca de que en no pocas oportunidades el pasaje de escenarios educativos autoritarios a democráticos puede producir cierta sensación de vacío, con la posibilidad de generar situaciones anárquicas. El respeto a normativas institucionales democráticas requiere una pedagogía también democrática que promueva alternativas a las prácticas educativas tradicionales.

Una de las publicaciones (Emmanuele, Boffa y Sanjurjo,1987) caracteriza las Asesorías Pedagógicas: a) forman parte de un nuevo proyecto ideológico político que prioriza la democratización en todos los niveles y el pluralismo científico e ideológico; b) conciben a la educación como una práctica social y como una problemática política sin visos de neutralidad; c) contribuyen a una revisión epistemológica de las ciencias de la educación; d) reemplazan la noción de práctica por la idea freireana de praxis que reúne la reflexión y la acción del hombre sobre el mundo para transformarlo; e) reemplazan la noción de perfeccionamiento docente por la reflexión sobre la propia práctica a través del taller de educadores; f) adhieren a una concepción dinámica y dialéctica del conocimiento científico como construcción histórico social y como unidad interdisciplinaria; g) reemplazan el aula, como foco de atención, por la institución, a los efectos de propiciar su desburocratización; h) el asesor pedagógico no es concebido como una figura externa a la problemática institucional, sino como un actor implicado en el devenir institucional.

\section{Epistemología y Educación}

La intención de los cuadernillos centrados en esta temática fue la de plantear algunos temas relacionados con el campo epistemológico que pudiesen aportar a la reflexión acerca de la tarea docente. En primer lugar, realizando una revisión crítica de sus supuestos epistemológicos y en segundo lugar, propiciando modalidades de "enseñanza/aprendizaje" alternativos. 
Se concibe al conocimiento como un proceso dialéctico que permite comprender y transformar la realidad, proceso mediante el cual los sujetos se comprenden y se transforman. Se trata de un conocimiento que no es absoluto ni independiente de la historia de las ciencias, en tanto es producido históricamente en el conjunto de las distintas prácticas sociales. No es lineal, ni continuo, ni neutral, sino dialéctico e histórico. Esta concepción de la epistemología y del conocimiento marca un corte con respecto a la etapa autoritaria, en la cual el conocimiento era presentado como algo inmutable, ahistórico, estático y compuesto por verdades absolutas.

Si bien la relación entre fundamentos epistemológicos y educación supone múltiples aspectos, la lectura de los cuadernillos revela la importancia otorgada al proceso de construcción del conocimiento. Se concibe al conocimiento como el producto de una práctica histórica y social, como un "interjuego dialéctico" entre lo individual y lo social, las estructuras cognitivas y la realidad exterior.

Se intenta mostrar, además, que en el acto educativo tanto quienes enseñan como quienes aprenden ponen en juego sus propias "matrices epistemológicas" y sus modelos de vinculación con el conocimiento que operarán inhibiendo o potenciando los aprendizajes. A partir de la pregunta ¿desde qué posición epistemológica hablan los docentes? comienzan a abrirse dos interrogantes que atraviesan el acto educativo: a) el objeto del conocimiento ¿es algo dado o construido?; b) el sujeto del conocimiento ¿es independiente de su contexto, de su historia o es un sujeto histórico, producto de múltiples prácticas sociales? Si la dictadura había generado una devaluación académica, en esta etapa la tarea consistió en establecer nuevas formas de legitimación del conocimiento (Monestés, 1987).

\section{Psicología y Educación}

La teoría psicoanalítica, que había sido objeto de censura durante la dictadura, se instala nuevamente en las carreras de Psicología y en el resto de las carreras humanísticas. La incorporación de contenidos referidos al psicoanálisis, en tanto refieren al sujeto que aprende, se produce rápidamente en la formación docente universitaria. Además de las obras de Freud y Lacan y de la re-edición de libros escritos en las dos décadas anteriores, comienzan a circulas nuevas producciones. La propuesta editorial de la Universidad Nacional de Rosario le dedica una Colección a Psicología y Psicoanálisis

Las principales nociones del psicoanálisis se introducen en la medida que dan cuenta de la constitución subjetiva del niño. Si la educación es una acción que se ejerce sobre los sujetos, resulta imprescindible conocer la estructura psicológica de los mismos. En ese sentido, el psicoanálisis ofrece la perspectiva de los procesos inconscientes y la psicología genética desarrollada por Piaget brinda elementos para conocer la inteligencia y las operaciones que la misma genera. De ahí que la psicología de la educación se presenta como un aporte 
fundamental para comprender mejor el proceso educativo y favorecer su desarrollo, "por cuanto su objeto son las condiciones subjetivas que producen y reproducen el aprendizaje o el estudio de los procesos psicológicos que están en juego en el aprendizaje y dan lugar a la operación educativa" (Ageno, 1988, p.7).

De este modo, la psicología de la educación queda estrechamente vinculada al aprendizaje y a las operaciones psíquicas que lo hacen posible. La relevancia otorgada a este tema se puede observar en las distintas propuestas curriculares de la época, donde esta temática forma parte de los marcos teóricos. Asimismo se destaca la importancia del rol del Psicólogo Educacional en tanto su intervención está relacionada con la problemática educativa, pero mirada desde el proceso que la hace posible, el aprendizaje, y desde todos los ámbitos donde ese proceso se produce: individual, grupal, institucional y comunitario.

\section{A modo de conclusión}

La propuesta editorial de la Universidad Nacional de Rosario en el periodo que nos ocupa sólo puede ser comprendida si se parte de los efectos que la dictadura y el terrorismo de Estado habían generado en la sociedad, en los sujetos, en las instituciones y en el campo educativo. Las universidades en general y la Universidad Nacional de Rosario en particular asumieron un firme compromiso con el proceso de democratización que se inaugura a fines de 1983. Ese compromiso alcanzó distintas modalidades. En el caso de la Universidad Nacional de Rosario una de ellas fue la publicación de estas Colecciones centradas en los distintos aspectos que se debían tener en cuenta en la tarea de educar en democracia.

Estas colecciones y cuadernillos dan cuenta del clima de época, de la preocupación de los actores universitarios por sostener y consolidar un sistema democrático no sólo a nivel político, sino también institucional. Estas publicaciones sin duda dejaron marcas en sus autores (la mayoría profesores de la Universidad de Rosario) en sus lectores (profesores y alumnos), en las aulas a través de debates y espacios de intercambio y en las estructuras y vínculos institucionales. En síntesis, se trató de una apuesta fuerte realizada por una institución universitaria comprometida con la democracia, la educación y el conocimiento.

\section{Notas Bibliográficas}

(1) Ministerio de Educación y Justicia, Secretaría de Educación (1985). "Lineamientos estratégicos para la democratización real de la educación". p. 21

(2) La Universidad Nacional de Rosario se creó el 29 de noviembre de 1968 como un desprendimiento de la Universidad Nacional del Litoral, a su vez creada en 1889 como universidad provincial bajo el nombre de Universidad de Santa Fe y nacionalizada en 1919. 
(3) Fragmento extraído del Discurso pronunciado por el Dr. Millet en el acto de asunción como Rector de la UNR, el día 21 de mayo de 1986. Disponible en la Dirección de Archivo, UNR.

(4) En esta etapa tanto el gobierno nacional como los gobiernos provinciales organizaron instancias de consulta a los docentes (denominadas Talleres, Mesas de Trabajo, etc.) con el objetivo de que participaran de los cambios curriculares correspondientes a las distintas jurisdicciones y niveles.

\section{Referencias Bibliográficas}

- $\quad$ Achili, E. (1987). La práctica docente: una interpretación desde los saberes del maestro. Cuadernos de Formación Docente № 1. Secretaría Académica, UNR.

- $\quad$ Ansaldi, W. (2006). Juego de patriotas. Militares y políticos en el primer gobierno posdictadura en Bolivia, Brasil y Uruguay. En Pucciarelli, A. (coord.) Los años de Alfonsín. ¿El poder de la democracia o la democracia al poder?, Buenos Aires: Siglo XXI.

- Bertoni, M.L. y Cano, D. (1990). La educación superior argentina en los últimos veinte años: tendencias y políticas. Propuesta Educativa No 2, FLACSO, Buenos Aires: Miño y Dávila.

- Braslavsky, C. (1989). La educación en la transición a la democracia: elementos y primeros resultados de una comparación. Propuesta Educativa № 1, FLACSO, Buenos Aires: Miño y Dávila.

- Buchbinder, P (2010). Historia de las universidades argentina, Buenos Aires: Sudamericana.

- Cano, D. (1985). La educación superior en la Argentina, Buenos Aires: Grupo Editor Latinoamericano.

- $\quad$ Coria, A. y Edelstein, G. (1993). El pedagogo: un discurso posible. Pensamiento Universitario No 1, Buenos Aires: Lembas.

- Delich, F. (1982). La construcción social de la legitimidad política en procesos de transición a la democracia. Crítica y Utopía nro. 9, Buenos Aires.

- $\quad$ Emmanuele, E. y Bertolano, L., (1988). Universidad, docencia y saber pedagógico. Cuadernos de Formación Docente № 6. Secretaría Académica, UNR.

- $\quad$ Emmanuele, E, Boffa, S. y Sanjurjo, L. (1987). La Asesoría Pedagógica: su espacio en el ámbito universitario. Colección Espacios $N^{\circ} 2$. Departamento de Formación Docente. Secretaría Académica UNR.

- Martínez Paz, F. (1986) El Sistema Educativo Nacional. Formación, desarrollo, crisis. Dirección Nacional de Publicaciones, Universidad Nacional de Córdoba.

- Monestés, M.C. (1987). Epistemología y Educación. Colección Espacios N 5. Departamento de Formación Docente. Secretaría Académica. UNR

- O’Donell, G (1982). El Estado Burocrático Autoritario. Buenos Aires: Ed. Belgrano.

- Vera, R. (1987). Orientaciones básicas de los Talleres de Educadores. Cuadernos de Formación Docente № 7. Secretaría Académica. UNR. 